\title{
BMJ Open Early-life exposure to air pollutants and adverse pregnancy outcomes: protocol for a prospective cohort study in Beijing
}

\author{
Jing Song, ${ }^{1}$ Yi Chen, ${ }^{1}$ Ling Wei, ${ }^{1}$ Ying Ma, ${ }^{1}$ Ning Tian, ${ }^{1}$ Shi Yun Huang, ${ }^{1}$ \\ Yin Mei Dai, ${ }^{1}$ Li Hong Zhao, ${ }^{1}$ Yuan Yuan Kong ${ }^{2}$
}

To cite: Song J, Chen Y, Wei L, et al. Early-life exposure to air pollutants and adverse pregnancy outcomes: protocol for a prospective cohort study in Beijing. BMJ Open 2017;7:e015895. doi:10.1136/ bmjopen-2017-015895

- Prepublication history for this paper is available online. To view these files please visit the journal online (http://dx.doi org/10.1136/bmjopen-2017015895).

Received 17 January 2017 Revised 23 June 2017 Accepted 27 July 2017

${ }^{1}$ Beijing Obstetrics and Gynecology Hospital, Capital Medical University, Beijing, China

${ }^{2}$ Beijing Maternal and Child Health Care Hospital, Capital Medical University, Beijing, China

Correspondence to Professor Yi Chen; bjfcyycy@sina.com

\section{ABSTRACT}

Introduction The association between early exposure to ambient air pollution and adverse pregnancy outcomes in China is unclear. This study will assess the risk of early-life exposure to air pollutants in Beijing and explore the viability of 8-hydroxydeoxyguanosine (8-OHdG) as a biological indicator to assess oxidative stress induced by early-life exposure to air pollution.

Methods and analysis Here, 2500 women with singleton pregnancies and their infants will be recruited from the Beijing Obstetrics and Gynecology Hospital. We will collect nine types of biological samples, including maternal serum, urine, placental tissue, umbilical cord tissue and umbilical cord blood during all three trimesters. The air pollution data (particulate matter (PM)2.5, PM10 and similar factors) will be recorded at official fixed-site monitoring stations closest to where the pregnant women live. We plan to assess the effect of air pollutants on adverse pregnancy outcomes and infant respiratory and circulatory disease using Cox regression and competitive risk analysis and explore possible critical windows of exposure during pregnancy using daily pollutant concentrations averaged over various periods of pregnancy combined with individual activity and physiological parameters. Maternal and umbilical cord blood samples (1000 samples) will be randomly selected for 8-OHdG assays to assess the correlation between exposures to air pollutants and oxidative stress. We will determine whether air pollutant exposure or 8-0HdG levels are associated with adverse pregnancy outcomes. SPSS and SAS statistical software will be used for data analysis. Cox regression and competing risk analysis will be used to compute the HR and population attributable risk.

Ethics and dissemination This research protocol has already been approved by the Medical Ethics Committee of Beijing Obstetrics and Gynecology Hospital. Written informed consent will be obtained from all study participants prior to enrolment. The results will be published in peer-reviewed journals or disseminated through conference presentations.

Trial registration number This study has been registered in WHO International Clinical Trial Register-Chinese Clinical Trial Registry under registration number ChiCTRR0C-16010181 (http://www.chictr.org.cn/showproj.aspx? proj=17328).

\section{INTRODUCTION}

Maternal and child health is the premise and basis of healthy development; it is also related

\section{Strengths and limitations of this study}

- This is the first birth cohort study to focus on the early-life exposure to air pollutants and adverse pregnancy outcomes in Beijing.

- This research aims to further investigate the mechanisms underlying the impact of air pollution to adverse birth outcomes.

- This study should provide a reference for future large-scale studies of early-life exposure to air pollutants in China.

- Restriction to a single city limits the generalisability of our research because the proportion of particulate matter (PM)2.5 from regional and local sources varies by geographic location.

- In this study, we consider age, socioeconomic predictors, daily activities and time spent indoors confounding factors. However, individual-level exposure will not be quantified by personal sampling. Quantification of an individual's exposure is imprecise without either a completely controlled environment or portable sampling equipment.

to the happiness and well-being of millions of households. Thus, people are making every effort to reduce the incidence of adverse pregnancy outcomes and neonatal morbidity. The rapid economic development and urbanisation of China have led to worsening trends in the overall quality of urban and regional environments. ${ }^{12}$ The impact of air pollution on human health has long attracted attention. Air pollution is directly linked to elevated mortality and morbidity through respiratory and cardiovascular diseases, and leads to adverse pregnancy outcomes. ${ }^{3-6}$ It affects infants directly and it can have a long-term impact on adult health, including inducing hypertension, coronary heart disease and non-insulin-dependent diabetes. ${ }^{7}$ The impact of air pollution on adverse pregnancy outcomes has been studied internationally for some time, and the epidemiological studies include cross-sectional surveys, follow-ups and cohort studies, ${ }^{8-10}$ which have strong 
abilities to demonstrate a causal relationship. Time series and case-crossovers have been used to study the impact of air pollution (particulate matter (PM) 2.5, $\mathrm{PM} 10, \mathrm{SO}_{2}$, $\mathrm{NO}_{2}$ and $\mathrm{O}_{3}$ ) on adverse pregnancy outcomes such as low birth weight, premature birth, birth defects, stillbirth and intrauterine inflammation, and maternal conditions such as pre-eclampsia and diabetes mellitus. ${ }^{11-16}$ In recent years, with the development of technology and cross-disciplinary approaches, individual maternal exposure to air pollutants is estimated by combining data from environmental monitoring stations and the nearby residential addresses of the mothers in question. An increasing number of studies have examined the effects of air pollution on adverse pregnancy outcomes, including studies in China. ${ }^{1718}$ According to the WHO air pollution database, China has higher levels of air pollution than Western countries. However, research into the effect of air pollution on adverse pregnancy outcomes in China has lagged and is quite limited. Environmental pollution studies have focused mainly on persistent organic pollutants, heavy metal pollution and atmospheric pollution. Most studies have used multivariate logistic regression or time series to analyse the relationship between exposure to atmospheric $\mathrm{PM} 10, \mathrm{SO}_{2}$ and $\mathrm{NO}_{2}$ during pregnancy and adverse pregnancy outcomes. The main adverse pregnancy outcomes studied have been preterm birth, birth defects and low birth weight. China's national environmental protection bureau monitored PM2.5 in 2013. There are only a few studies directly linking PM2.5 to long-term health effects in China. Given the large number of births in China, the impact of air pollution on adverse pregnancy outcomes must not be disregarded. However, studies on this issue in China are relatively limited. It is therefore necessary to conduct large-sample prospective cohort studies to quantitatively evaluate individual exposure and to analyse the impact of air pollution on birth outcomes and explore the biological mechanisms.

\section{OBJECTIVES}

\section{Primary objectives}

The primary aims of this study are to establish a maternal and birth cohort, then set up a maternal and newborn information database and biological sample library based on that cohort.

We will record the air pollutant indicators published daily by the Environmental Monitoring Station near the pregnant women's residences, including PM2.5, PM10, $\mathrm{SO}_{2}$ and $\mathrm{NO}_{2}$ at different stages of pregnancy.

The purpose of this work is to study the correlations between environmental exposure early in life and adverse pregnancy outcomes and to investigate the correlations between different levels of exposure to ambient air pollution at different stages of pregnancy and adverse pregnancy outcomes including preterm birth, fetal growth restriction, low birth weight and the correlations to birth defects like congenital heart disease, neural tube defects, cleft lip and palate, and similar conditions.

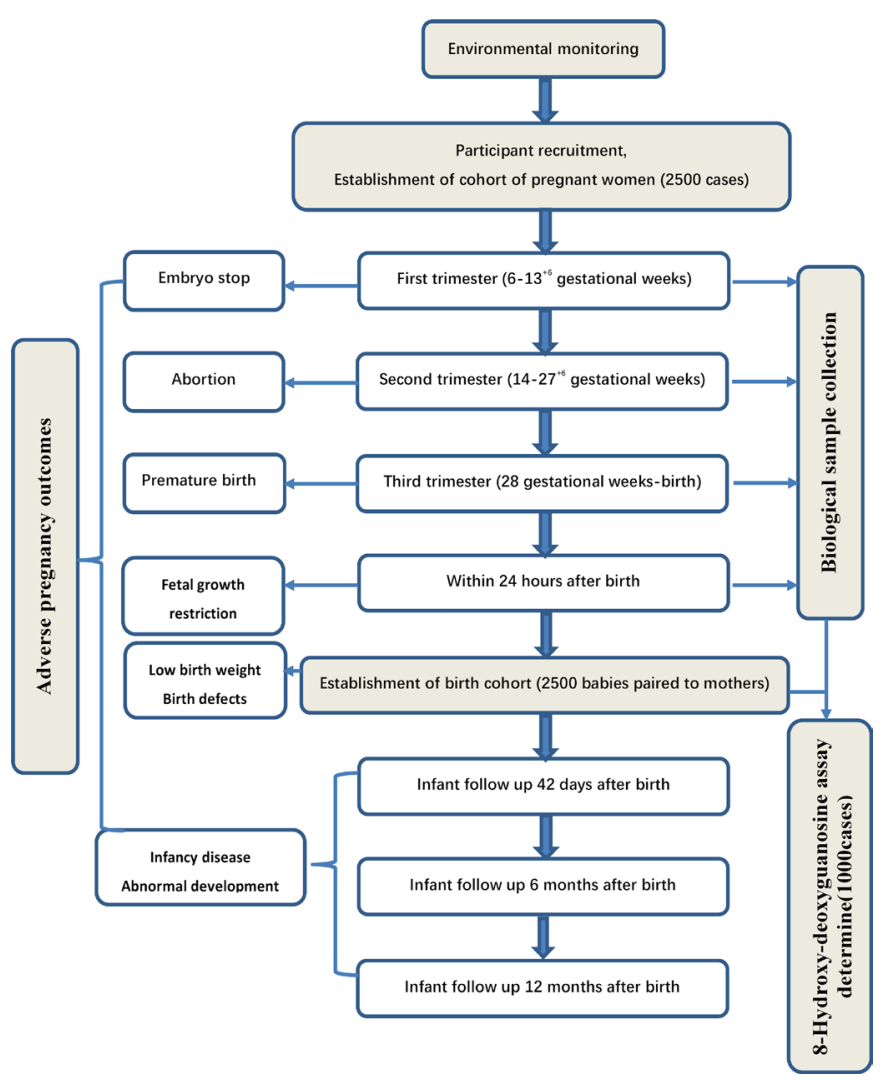

Figure 1 Flow chart of the study.Early-life exposure to air pollutants and adverse pregnancy outcomes.

\section{Secondary objectives}

To explore the significance of using 8-hydroxydeoxyguanosine $(8-\mathrm{OHdG})$ as a biological indicator to assess early-life exposure to air pollutants and investigate the mechanisms underlying these adverse outcomes.

\section{STUDY DESIGN}

\section{Study population and cohort design}

This is a prospective cohort study. A total of 2500 singleton pregnant women will be recruited from 1 January 2017 to 31 December 2018 during their routine antenatal check at the Beijing Obstetrics and Gynecology Hospital of Capital Medical University. They will undergo delivery in the hospital and must plan to reside in Beijing for at least the next 3 years. They must provide informed consent and agree for their newborns to participate in the birth cohort for a 1-year follow-up. This research is performed to establish a maternal and birth cohort and biological sample library studying covering correlations between exposure to air pollutants early in life and adverse pregnancy outcomes. A flow chart of this trial is given in figure 1.

\section{Exclusion criteria}

Each patient's history of contact with radioactive material during early pregnancy, history of use of any teratogenic drug or fever during early pregnancy (body temperature $>38.5^{\circ} \mathrm{C}$ ); family history of genetic disease; 
inbreeding; and chromosome abnormalities of pregnant women or their husbands are to be recorded.

\section{Data collection and management}

\section{Estimated sample size}

We refer to incidence of adverse outcome and risk ratio(RR) value under exposure reported in the literature and use this formula to calculate the sample size. The estimated sample size is no more than 2500 cases with $90 \%$ statistical power.

$$
n=\frac{\left(Z_{\alpha} \sqrt{2 \overline{p q}}+Z_{\beta} \sqrt{p_{0} q_{0}+p_{1} q_{1}}\right)^{2}}{\left(p_{1}-p_{0}\right)^{2}}
$$

\section{Collection of participant data}

Pregnant women will be recruited according to the inclusion criteria. After providing written informed consent, they will fill out the questionnaires with the help of trained investigators. The maternal population's financial information, detailed residential history going back 3 months before pregnancy and through early pregnancy, health condition, lifestyle, history of pregnancy, anamnesis, mode of conception and condition during early pregnancy will be noted. Detailed individual information collected from pregnant women (through a questionnaire, detailed in Questionnaires): age, height, weight, body mass index, occupation (occupational exposure), education and other demographic data. Detailed residential address information, daily outdoor activities and time spent outdoors, outdoor exertion and protective measures used during smog episodes, usage of air purifying apparatus, and cooking habits. Health condition before pregnancy, menstrual history, anamnesis and pregnancy history, mode of conception, history of smoking or passive smoking, alcohol consumption, lifestyle during the 3 months preceding pregnancy, history of adverse environmental exposure, dietary habits, folic acid intake, morning sickness, mental health, and some information regarding the husband, including height, weight, occupation, smoking and alcohol assumption will be recorded. Everyone's information will be entered into the database and numbered, and each infant will be assigned a number matching his or her mother's. The pregnant women will enter the cohort early during pregnancy and be followed throughout early pregnancy $\left(6-13^{+6}\right.$ gestational weeks), the second trimester $\left(14-27^{+6}\right.$ weeks), the third trimester ( $>28$ weeks), birth, 42 days after birth, and the neonatal and infant stages; information including clinical data such as clinical phenotype, laboratory results, ultrasound images, treatment and prognosis, and infant growth data will be systematically collected. Newborns will enter the birth cohort upon birth. A medical database with 2500 pairs of mothers and infants covering the entire process through pregnancy and infancy will be established. Outcome measurements will be checked by a clinical expert. These data will be written on the case report form (CRF) by a certificated clinical research coordinator.

\section{Biological sample collection}

Biological samples will be collected based on the establishment of the maternal and birth cohorts. Biological sample libraries containing maternal serum, urine collected during the first trimester $\left(1-13^{+6}\right.$ weeks $)$, second trimester $\left(14-27^{+6}\right.$ weeks), third trimester (28 weeks to delivery) and at birth will be established. Placental tissue, umbilical cord tissue, umbilical cord blood, meconium, children's dried blood spots, maternal hair and colostrum/breast milk will be collected after delivery. Chorionic Villus Sampling(CVS)samples and amniotic fluid samples $(10 \mathrm{~mL})$ will be collected from cases who receive prenatal diagnosis of fetal malformations found. A total of more than 35000 samples are expected.

Maternal peripheral venous blood $(5 \mathrm{~mL})$ and urine $(5 \mathrm{~mL})$ will be collected during early pregnancy, the second trimester, the third trimester and 24 hours after birth. Serum and urine will be separated and cryopreserved at $-80^{\circ} \mathrm{C}$. CVS samples and amniotic fluid samples $(10 \mathrm{~mL})$ will be collected from cases who received a prenatal diagnosis of fetal malformations during the second trimester.

Umbilical cord blood $(5 \mathrm{~mL})$, the umbilical cord itself $(10 \mathrm{~cm})$ and $2 \times 2 \times 2 \mathrm{~cm}$ full-thickness placental samples (rinsed in ice-cold saline to remove blood) will be collected and cryopreserved at $-80^{\circ} \mathrm{C}$ at the time of birth. Then $5 \mathrm{~mL}$ of maternal urine, a strand of hair $3 \mathrm{~mm}$ in length and maternal colostrum/breast milk $(1 \mathrm{~mL})$ will be collected 24 hours after birth and cryopreserved in the library. The delivery process and newborn's condition will be recorded in detail, and the survey questionnaire will be completed by the physician. A detailed birth index will be recorded. It will include gestational week, infant gender, weight, length, Apgar score, neonatal complications, and appearance and organ abnormalities. Each baby's meconium will also be collected to examine metabolites and assess the faecal microbiota. Dried blood spot specimens in one heel of each newborn will be collected onto specially manufactured absorbent filter paper and stored in plastic bags.

\section{Infancy visits}

An early growth monitoring system will be established based on the maternal and birth cohort and newborns will be included starting at birth. Anthropometric measurements and questionnaires related to feeding and sickness will be conducted at a community healthcare centre when the children are 42 days, 6 months and 12 months old. At 42 days after birth, the mother will bring the newborn to the health centre. Information pertaining to infant growth, development and breastfeeding will be recorded, and the survey questionnaire will be completed by the physician. At 6 and 12 months after birth, the infant will be brought to the community healthcare clinic for anthropometric measurements and evaluation of its development. Infant development will be monitored, and height, weight, head circumference and number of teeth will be determined, and questionnaires will be completed 
regarding feeding and illnesses, including diseases of the respiratory or cardiovascular system as diagnosed by doctors.

\section{Air pollution data collection for exposure assessment}

There are 35 air quality monitoring stations distributed in the different districts in Beijing, which is in line with national air quality monitoring position with the requirement of technical specification. The air pollution level and population exposure will be evaluated using environmental monitoring data (PM2.5, $\mathrm{PM} 10, \mathrm{SO}_{2}, \mathrm{NO}_{2}$ and $\mathrm{O}_{3}$ ) published daily by the Beijing Municipal Environmental Monitoring Center. Data will be collected from the closest monitoring station where pregnant women live. Daily meteorological data such as temperature and humidity will also be collected from the national meteorological centre and used as a confounding control in the analysis. We plan to explore potential critical windows of exposure during pregnancy using daily PM2.5, PM10 and other pollutant concentrations averaged over various periods, including the 3 months before pregnancy and each of the three trimesters of pregnancy. Exposure throughout the entire pregnancy will be calculated as the mean of all days of the pregnancy.

\section{Recording of outcome measures}

Adverse pregnancy outcomes include birth defects, preterm birth, low birth weight and fetal growth restriction. Associated birth defects include anencephaly, neural tube defects (anencephaly, spina bifida, congenital spina bifida, congenital hydrocephalus), cleft lip and palate (cleft palate, cleft lip, cleft lip with cleft palate), ear deformity (microtia including anotia, outer ear and other malformations), gastrointestinal abnormalities (oesophageal atresia or stenosis, anorectal atresia or stenosis (including no anus)), urinary tract malformations (hypospadias, bladder exstrophy), abdominal malformation (congenital diaphragmatic hernia, omphalocele, gastroschisis), Down syndrome (trisomy 21), congenital heart disease, limb malformations (varus clubfoot, polydactyly, syndactyly and longitudinal deficiency), limb shortening and ambiguous genitalia. Preterm birth refers to birth at a gestational age of $28-36^{+6}$ weeks (gestational age is calculated according to the last menstrual period combined with an early pregnancy ultrasound image). Low birth weight refers to birth weight below $2500 \mathrm{~g}$.

\section{Data analysis}

Investigate the relationship between adverse air pollutant exposure of the population and offspring development outcomes (specifically adverse pregnancy outcomes)

Data will be analysed to evaluate the association between air pollutant exposures and pregnancy outcomes. All study participants will be closely followed up throughout the pregnancy, and pregnancy complications (such as pregnancy-induced hypertension, gestational diabetes, fetal growth restriction, premature rupture of membranes and intrauterine infection) will be recorded in detail in a timely manner. Based on PM2.5, PM10, $\mathrm{SO}_{2}, \mathrm{NO}_{2}$ and $\mathrm{O}_{3}$ concentrations and on many kinds of calculation methods, such as the closest point method and inverse distance weighting, combined with the individual activity patterns and physiological parameters, we will calculate the HR and population attributable risk (PAR) using Cox regression and competitive risk analysis in order to assess the effect of air pollutants on adverse pregnancy outcomes.

Investigate the relationship between early-life exposure and oxidative stress in pregnant women and fetuses

Studies showed that exposure to particulate air pollution early in life plays a role in increasing systemic oxidative stress. Oxidative stress is believed to be involved in the mechanisms underlying the effects of air pollution on human health. 8-hydroxy-2'-deoxyguanosine (8-OHdG) is a sensitive indicator of oxidative stress. We will investigate 8-OHdG levels during different time windows in maternal blood and cord blood and explore the relationship between exposure to air pollutants and oxidative stress. Maternal and umbilical cord blood samples (1000 each) will be randomly selected from the cohort for 8-OHdG assay to determine the correlation of earlylife exposures to oxidative stress in pregnant women and fetuses. All recruited pregnant women paired with their babies will be numbered starting at 1 , and then either odds or evens will be randomly selected for testing.

\section{Quality control}

\section{Data management}

The questionnaires were designed based on extensive reviews of the literature and advice from obstetrics and paediatrics experts. Detailed standards will be established for subject recruitment of the maternal and birth cohort. The exact addresses of the pregnant women will be recorded to allow selection of the closest environmental monitoring stations. Each data monitoring centre is in Beijing Obstetrics and Gynecology Hospital. The participants may drop out of the trial at any time for any reason. The physician should assess each patient and provide any needed assistance. Incidences of loss to follow-up will be reported to the chief designer of this trial weekly.

\section{Investigator training}

A research manual (CRF tables) will be prepared to standardise the investigation and survey techniques and to improve the working ability and responsibility of investigators; specialised physicians will be responsible for the clinical diagnostic reports.

\section{Questionnaire verification}

Clinical physicians will check the questionnaires after every survey for logical errors and missed information, and additional surveys will be conducted within 24 hours to supplement the data. 


\section{Data entry}

A combination of hard copy questionnaires and computer database entries will be used for data collection. Concurrent validity data will be collected for quality control. Training will be provided to all personnel involved in data entry beforehand. Two specialised personnel will be responsible for the second round of quality control assessment every month.

\section{Development of a protocol for biological sample collection}

Sample collection will be performed in strict accordance with protocol, and the rate of loss to follow-up will be closely controlled. Biological sample libraries will be managed by assigned individuals, Standard Operating Procedure(SOP) regulations will be developed, and sample collection site size, standing time, transportation and storage will be strictly controlled according to the protocol. To ensure data accuracy, completeness and clarity, all samples will be stored using the sample number matching the maternal information and recorded and managed via computer management software system, which will be logged with the hospital information system(HIS) and regularly exported for storage.

\section{Adverse events}

All adverse events in any pregnant woman or infant will be recorded in detail and closely monitored until stabilisation or resolution. The chief designer of this trial will be informed immediately and responsible for reporting to Scientific Research Department and Medical Ethics Committee of Beijing Obstetrics and Gynecology Hospital. Regular monitoring will be used to ensure that all adverse events are identified and addressed appropriately.

\section{Statistical analysis}

Epidata V.3.1 software will be used for double entry of the data and logic verification to establish a database; SPSS V.18.0 (SPSS) and SAS V.9.3 (SAS Institute) statistical software will be used for data analysis. Descriptive analysis will be performed first to compute the general maternal and infant demographic data and distribution of birth outcomes and birth defects. Cox regression and competing risk analysis will be used to compute the HR and PAR and to estimate the effect of main air pollutants on major adverse birth outcomes (low birth weight, preterm birth and fetal growth restriction) and birth defects (congenital heart disease). Pearson correlation coefficients and multiple linear regression will be used to assess the association between air pollutant exposure and $8-\mathrm{OHdG}$ levels. We will then determine whether PM exposure or $8-\mathrm{OHdG}$ levels are associated with adverse pregnancy outcomes.

\section{DISCUSSION}

Epidemiological studies in Europe and the USA have shown that exposure to air pollution during pregnancy is associated with adverse pregnancy outcomes. ${ }^{19-21}$ With
China's rapid development, air pollution has become more serious than in previous years, especially in Beijing. ${ }^{22}$ The average PM2.5 concentration hit $127.1 \mu \mathrm{g} /$ $\mathrm{m}^{3}$ in December 2016 in Beijing, which is higher than the current US EPA National Ambient Air Quality Standard of $12 \mu \mathrm{g} / \mathrm{m}^{3}$. However, only a few studies have directly linked PM2.5 to pregnancy outcomes in China. It is not clear whether premature birth, low birth weight, congenital anomalies, other adverse maternal outcomes, or infant respiratory or circulatory issues are significantly associated with environment exposure. This study will establish a birth cohort in Beijing and determine whether there are any associations between prenatal exposure to air pollution and adverse pregnancy outcomes. This work will explore the mechanisms underlying the impact of early-life exposure on adverse pregnancy outcomes. $\mathrm{PM}$ air pollution exposure is a known risk factor for the induction of inflammatory and oxidative stress response during pregnancy. ${ }^{23}$ Maternal exposure to air pollutants might exacerbate oxidative damage and generate reactive oxygen species that would induce DNA damage. Most studies indicate $8-\mathrm{OHdG}$ is a biomarker for oxidative stress, and exposure to air pollution early in life may play a role in increasing systemic oxidative stress, at levels of $8-\mathrm{OHdG}$ in both mother and child. ${ }^{24}{ }^{25}$ We will measure the 8-OHdG levels of the mothers and babies to explore the relationship between exposure to air pollutants during different time windows in pregnancy and oxidative stress. We plan to determine whether 8-OHdG levels or exposure to air pollutants is associated with adverse pregnancy outcomes and infant respiratory and cardiovascular diseases. We will attempt to determine the mechanisms underlying these adverse outcomes caused by air pollutant factors.

Acknowledgements The authors would like to thank the project sponsors, Professor Zhi Wei Sun and Professor Xian Qing Zhou, both of Capital Medical University. We would like to thank all subjects who have agreed to participate in the study and the staff at the Beijing Obstetrics and Gynecology Hospital, Capital Medical University and Beijing Maternal and Child Health Care Hospital.

Contributors YC is the chief designer of this trial. JS, LW, YM, NT, SYH, YMD, LHZ and YYK are coinvestigators. YMD assisted in designing the study and developed the objectives and methods of the study. JS and LW drafted the manuscript, and YC was responsible for supervision of the study and revision of the manuscript. All authors have contributed to the composition of the study protocol and have approved the final version of this manuscript. YC, JS, LW, YM and NT were involved in the recruitment of patients. SYH was responsible for the collection of clinical data for pregnant women. LHZ was responsible for the management of biological sample library. YYK was responsible for neonatal follow-up.

Funding This work is a part of a study at the School of Public Health at the Beijing Key Laboratory of Epidemiology at Capital Medical University (a comprehensive study of the effects of air pollution on cardiopulmonary disease in Beijing: exposure science, toxicology and environmental epidemiology) provided by Professor Zhi Wei Sun, with the support of China's National Natural Science Foundation Program grant funding (81571130090).

Competing interests None declared.

Patient consent Obtained.

Ethics approval The protocol has been approved by the Medical Ethics Committee of Beijing Obstetrics and Gynecology Hospital, Capital Medical University. Written informed consent was obtained from all study participants prior to enrolment in the study. 
Provenance and peer review Not commissioned; externally peer reviewed.

Open Access This is an Open Access article distributed in accordance with the Creative Commons Attribution Non Commercial (CC BY-NC 4.0) license, which permits others to distribute, remix, adapt, build upon this work non-commercially, and license their derivative works on different terms, provided the original work is properly cited and the use is non-commercial. See: http://creativecommons.org/ licenses/by-nc/4.0/

(C) Article author(s) (or their employer(s) unless otherwise stated in the text of the article) 2017. All rights reserved. No commercial use is permitted unless otherwise expressly granted.

\section{REFERENCES}

1. Li G, Fang C, Wang S, et al. The effect of economic growth, urbanization, and industrialization on fine particulate matter (PM2.5) concentrations in China. Environ Sci Technol 2016;50:11452-9.

2. Lu X, Lin C, Li Y, et al. Assessment of health burden caused by particulate matter in Southern China using high-resolution satellite observation. Environ Int 2017;98:160-70.

3. Guo $\mathrm{Y}$, Zeng $\mathrm{H}$, Zheng $\mathrm{R}$, et al. The association between lung cancer incidence and ambient air pollution in China: a spatiotemporal analysis. Environ Res 2016;144:60-5.

4. DeFranco E, Moravec W, Xu F, et al. Exposure to airborne particulate matter during pregnancy is associated with preterm birth: a population-based cohort study. Environ Health 2016;15:2-8.

5. Olsson D, Mogren I, Forsberg B. Air pollution exposure in early pregnancy and adverse pregnancy outcomes: a register-based cohort study. BMJ Open 2013;3:e001955.

6. Andersen ZJ, Sram RJ, Ščasný M, et al. Newborns health in the Danube region: environment, biomonitoring, interventions and economic benefits in a large prospective birth cohort study. Environ Int 2016;88:112-22.

7. Thiering E, Cyrys J, Kratzsch J, et al. Long-term exposure to traffic-related air pollution and insulin cohort. Diabetologia 2013;56:1696-704.

8. Vrijheid M, Slama R, Robinson O, et al. The human early-life exposome (HELIX): project rationale and design. Environ Health Perspect 2014;122:535-44.

9. Lamichhane DK, Leem JH, Lee JY, et al. A meta-analysis of exposure to particulate matter and adverse birth outcomes. Environ Health Toxicol 2015;30:e2015011.
10. Gray SC, Edwards SE, Schultz BD, et al. Assessing the impact of race, social factors and air pollution on birth outcomes: a populationbased study. Environ Health 2014;13:4.

11. Laurent $\mathrm{O}, \mathrm{Hu} \mathrm{J}$, Li L, et al. Sources and contents of air pollution affecting term low birth weight in Los Angeles County, California 2001-2008. Environ Res 2014;134:488-95.

12. Stieb DM, Chen L, Eshoul M, et al. Ambient air pollution, birth weight and preterm birth: a systematic review and meta-analysis. Environ Res 2012;117:100-11.

13. Lavigne E, Yasseen AS, Stieb DM, et al. Ambient air pollution and adverse birth outcomes: differences by maternal comorbidities. Environ Res 2016;148:457-66.

14. Nachman RM, Mao G, Zhang X, et al. Intrauterine inflammation and maternal exposure to ambient PM2.5 during preconception and specific periods of pregnancy: the Boston birth cohort. Environ Health Perspect 2016;124:1608-15

15. Green R, Sarovar V, Malig B, et al. Association of stillbirth with ambient air pollution in a California cohort study. Am J Epidemiol 2015;181:874-82.

16. Nieuwenhuijsen MJ, Dadvand P, Grellier J, et al. Environmental risk factors of pregnancy outcomes: a summary of recent meta-analyses of epidemiological studies. Environ Health 2013;12:6.

17. Zhao N, Qiu J, Zhang Y, et al. Ambient air pollutant PM10 and risk of preterm birth in Lanzhou, China. Environ Int 2015;76:71-7.

18. Qian Z, Liang S, Yang S, et al. Ambient air pollution and preterm birth: a prospective birth cohort study in Wuhan, China. Int J Hyg Environ Health 2016;219:195-203.

19. Fleisch AF, Kloog I, Luttmann-Gibson H, et al. Air pollution exposure and gestational diabetes mellitus among pregnant women in Massachusetts: a cohort study. Environ Health 2016;15:40.

20. Schembari A, de Hoogh K, Pedersen M, et al. Ambient air pollution and newborn size and adiposity at birth: differences by maternal ethnicity (the born in Bradford study cohort). Environ Health Perspect 2015;123:1208-15.

21. Beelen R, Raaschou-Nielsen O, Stafoggia M, et al. Effects of longterm exposure to air pollution on natural-cause mortality: an analysis of 22 European cohorts within the multicentre ESCAPE project. Lancet 2014;383:785-95.

22. Lin C, Li Y, Lau AKH, et al. Estimation of long-term population exposure to PM 2.5 for dense urban areas using 1-km MODIS data. Remote Sens Environ 2016;179:13-22.

23. Mazzoli-Rocha F, Fernandes S, Einicker-Lamas M, et al. Roles of oxidative stress in signaling and inflammation induced by particulate matter. Cell Biol Toxicol 2010;26:481-98.

24. Grevendonk L, Janssen BG, Vanpoucke C. et, al. Mitochondrial oxidative DNA damage and exposure to particulate air pollution in mother-newborn pairs. Environmental Health 2016;20:1-8.

25. Svecova V, Rossner P, Dostal M, et al. Urinary 8-oxodeoxyguanosine levels in children exposed to air pollutants. Mutat Res 2009;662:37-43. 